\title{
Impacts of COVID-19 lockdowns on frailty and wellbeing in older people and those living with long-term conditions
}

\author{
Carol Holland ${ }^{1, A-F}$, Ian Garner, 1,B,C,E, Jane Simpson ${ }^{1, A-C, E, F, F}$, Fiona Eccles ${ }^{1, A-C, E, F}$, \\ Esperanza Navarro Pardo $2, A, B, C, E, F$, , Calum Marr ${ }^{1, A, B, C, E, F}$, Sandra Varey ${ }^{1, A, B, E, F}$ \\ ${ }^{1}$ Centre for Ageing Research, Lancaster University, UK \\ 2 School of Psychology, University of Valencia, Spain \\ A - research concept and design; $B$ - collection and/or assembly of data; $C$ - data analysis and interpretation; \\ $\mathrm{D}$ - writing the article; $\mathrm{E}$ - critical revision of the article; $\mathrm{F}$ - final approval of the article
}

Address for correspondence Carol Holland

E-mail: c.a.holland@lancaster.ac.uk

Funding sources

None declared

Conflict of interest

None declared

Received on November 9, 2021

Accepted on November 22, 2021

Published online on November 25, 2021

Cite as

Holland C, Garner I, Simpson J, et al. Impacts of COVID-19 lockdowns on frailty and wellbeing in older people and those living with long-term conditions. Adv Clin Exp Med. 2021;30(11):1111-1114. doi: 10.17219/acem/144135

DOI

10.17219/acem/144135

Copyright

Copyright by Author(s)

This is an article distributed under the terms of the

Creative Commons Attribution 3.0 Unported (CC BY 3.0)

(https://creativecommons.org/licenses/by/3.0/)

\begin{abstract}
Lockdowns and social distancing have been important and successful strategies to limit the spread of the coronavirus disease 2019 (COVID-19) virus. However, excess deaths related to non-COVID-19 causes have been reported, suggesting issues around availability and use of health services, particularly for people with conditions needing ongoing medical support. In addition, evidence indicates that a range of age-related diseases and frailty are impacted by physical activity and social engagement, both limited in lockdown situations. It is therefore important to learn from the effects of lockdowns in order to limit any impacts, while still protecting people from the infection. This editorial summarizes two research themes at the Centre for Ageing Research at Lancaster University in the UK, one assessing impacts of lockdown for people living with a long-term neurodegenerative condition, Parkinson's disease, and one assessing longitudinal impacts on frailty and wellbeing, with older adults aged over 70, including those living with at least one long-term condition. Uncertainty related to Parkinson's disease and to COVID-19 amplified each other, and cancelled clinical appointments and limitations on physical activity had very significant impacts on wellbeing for this group. In the longitudinal study, frailty was more severe during lockdown periods. While lockdowns reduce spread of the virus, becoming frailer could make older adults more vulnerable to the effects of the virus during these periods. Regular exercise during lockdown had beneficial effects aiding recovery once restrictions relaxed. These studies suggest factors that could lessen negative impacts of future lockdowns. Maintaining physical activity and providing access to health services during periods of lockdown are suggested as priorities.
\end{abstract}

Key words: COVID-19, physical distancing, Parkinson's disease, frailty, aged 
More than 5 million people worldwide have now died of the coronavirus disease 2019 (COVID-19) virus, with many countries also beginning to report excess deaths (deaths in excess of what would be expected for the time of year based on pre-pandemic years) from other causes, related to stretched medical resources or fear of attending hospitals. The World Health Organization (WHO) survey, ${ }^{1}$ using data from 105 countries, found that the treatment of noncommunicable conditions had been disrupted by the pandemic, particularly the treatment of hypertension, diabetes, cancer, and cardiovascular emergencies. Non-COVID-19 mortality represents between one and two thirds of the excess mortality in different countries, ${ }^{2-4}$ and its causes include heart disease, diabetes, dementia and other causes. This has been attributed to a significant drop in use of healthcare; for example, in Greece, primary care visits dropped by $24.8 \%$ and hospital surgical procedures by $23.1 \%$, suggesting that the success of lockdowns in reducing infections and deaths from COVID-19 had come at the expense of non-COVID-19 patients, leaving a significant amount of unmet need in the population. ${ }^{4}$

Work to examine impacts of lockdowns on populations is crucial if we are to learn what works compared to what increases risks and healthcare burdens in the longer term. This is specifically the case for older age groups, given age-related increase in frequency of long-term conditions that need regular and ongoing healthcare support. ${ }^{5}$ The greater impact of COVID-19 infection on older adults, particularly those with existing long-term conditions is clear ${ }^{6}$ with respiratory issues, morbid obesity, dementia, diabetes, atrial fibrillation, and hypertension, all being risk factors for COVID-19-related hospitalizations. ${ }^{7,8}$ Frailty also increases with age, which can be associated with multiple chronic diseases but can also occur in the absence of specific diseases. Frailty is defined as a state of increased vulnerability to negative outcomes ${ }^{9}$ and in line with this definition, the outcomes of COVID-19 infection are more severe for frail older adults. ${ }^{10,11}$ Therefore, lockdown and social distancing strategies are vital to protect older adults. Moreover, we need to determine what impacts such strategies may have.

It is thus important to understand how lockdown restrictions may have affected older people living with, and without, long-term conditions, but also how they may have influenced frailty levels, and so vulnerability to infection, over the course of the pandemic to date. Physical and social inactivity caused by lockdown may be expected to have both an immediate and lasting effect on older adults' health, given that low exercise and social engagement have been associated with an increased risk of frailty, cognitive decline and dementia. ${ }^{12-14}$ Many older adults reported a significant reduction in normal activities they do to promote healthy ageing during lockdown - e.g., in a Spanish study, $65.7 \%$ reported doing less physical activity. ${ }^{15}$ Therefore, it is important to consider what factors may mitigate adverse effects of lockdown on older adults and those living with long-term conditions, with coping resources, and health behaviors such as physical and social engagement identified as candidate protective factors. Our own work has begun to examine some of these issues, first in a group of people living with a long-term neurodegenerative condition, Parkinson's disease, and second in groups of people aged over 70, half of whom living with multiple long-term conditions.

Our qualitative work with people living with Parkinson's ${ }^{16}$ focused on the uncertainty related to living with the illness in a time of pandemic and lockdowns. Four specific themes were extracted from the interview data: (1) COVID-19 is amplifying existing fears and difficulties around the uncertainty of Parkinson's, including those relating to hospitalization, loss of function and independence, accessibility of medication, and worries about treatment if they did get a COVID-19 infection. Many participants also realized that the two sources of uncertainty (their chronic, neurodegenerative illness and COVID-19) amplified each other; (2) Practical and psychological efforts to manage uncertainty. Participants attempted to gain control of the uncertainty of the situation. Their approach to the additional uncertainty created by social restrictions was pragmatic and focused on acceptance. The psychological toll of dealing with COVID-19 against a backdrop of also managing a serious health condition was acknowledged by the participants; (3) Benefit-finding as a way of acknowledging the positive aspects of lockdown: Despite multiple challenges, participants' ability to see the positives was important in balancing the potential psychological distress from having to manage an additional set of stressors, by reassessing priorities and feeling an increase in personal resilience; (4) Risk and future management in the context of uncertainty: While some of the participants' fears related to contracting COVID-19, similar levels of anxiety were also expressed about lockdown causing permanent effects on their health and wellbeing, e.g., due to cancelled health appointments or lack of availability of physical and social activity.

A key learning point was that the clinical appointments being cancelled during the pandemic was particularly worrying, given evidence on the role of healthcare communication and support in reducing illness uncertainty and communicating hope, especially during healthcare crises. ${ }^{17}$ Supporting this, Parkinson's UK report, based on a large quantitative survey, also found loss of physical exercise was a problem, due to its impact on both physical and mental wellbeing and significant problems with loss of medical appointments. ${ }^{18}$

In our second study (unpublished data), potential changes in physical and mental wellbeing in older adults were assessed across a 12-month period through lockdown variations to determine how frailty may change in relation to restrictions, focusing on a multidimensional approach to frailty and its measurement. ${ }^{19}$ We also aimed to consider if coping resources mitigate any deterioration in health and 
wellbeing associated with lockdown, and how frequency of physical activity and experienced social isolation may influence change in frailty, loneliness, quality of life, and perceptions of environmental age-friendliness (physical and social accessibility, community support and outreach).

Seventy participants were recruited in England and Spain, time periods illustrated in Fig. 1. While lockdowns were similar between the countries, the first lockdown, during which we conducted our first assessment (our Time 1), was stricter in Spain with people not being allowed out just for a walk, while in the UK residents were encouraged to take daily exercise. Also, at the time of our fourth assessment (Time 4), restrictions were increasing again in the regions of England where our participants were located. The last assessment was in June 2021 by which time all participants had been vaccinated.

Volunteers, all living independently, were aged over 70, with 50 participants in England and 20 in Spain. Overall, 24 participants were considered 'high risk' as determined using the UK National Health Service (NHS) lists of highrisk conditions, ${ }^{20}$ meaning they had at least one long-term health condition that made them more vulnerable to severe effects of COVID-19.

Assessments included measures of: multidimensional frailty; quality of life; loneliness; age-friendliness of their environment; physical activity (days a week they engaged in at least $30 \mathrm{~min}$ of exercise); social contact; coping resources (what things were helping them cope during the pandemic, e.g., family support, community groups or watching TV).

Results showed that the average frailty was highest at the time of the first assessment when restrictions were at their most stringent (it was higher in Spain), then decreased in severity in both countries to the third assessment, representing a time period with lower restrictions. Among the Spanish group, frailty continued to decrease but in the English group, frailty rose again at the fourth assessment, corresponding to a period in which our participants were subject to the stricter of the UK "tiered" lockdowns and to the beginning of the darker evenings and harsher winter than expected in Spain. At our final assessment, one year after the first, all participants had received two doses of a COVID-19 vaccine and social distancing had largely eased. At this point, frailty was significantly lower than in the strict first lockdown.

We also found changes in how participants viewed their environment. Across both countries, participants seemed to view their environment as more age-friendly, more able to meet their needs, e.g., for access to general practitioner (GP) surgeries or social engagement, when fewer lockdown restrictions were in place.

A year after the first lockdown, participants viewed their environment as significantly more age-friendly than at the beginning. We did not find any evidence to suggest that quality of life or loneliness showed significant changes over time, but we did find an impact of level of physical exercise on changes in frailty. Participants who engaged in exercise more frequently during lockdown showed a greater decrease in frailty severity as restrictions were eased. Having more or fewer coping resources did not seem to affect changes in frailty in this study.

The key learning points are:

(i) Frailty in older adults, including those with underlying health conditions that put them at higher risk

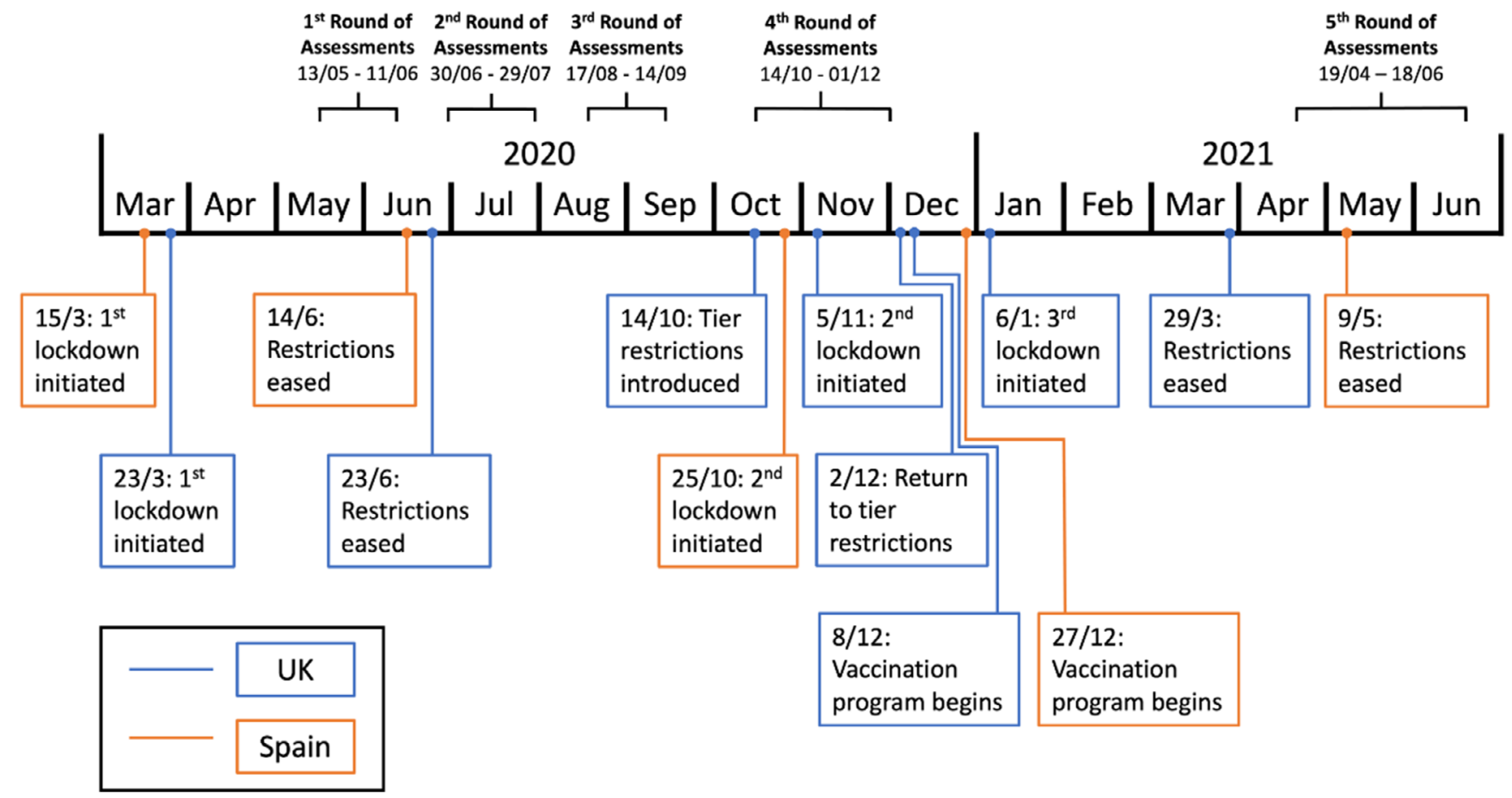

Fig. 1. Study timeline 
of COVID-19 infection, was more severe during periods of lockdown compared to when restrictions were eased. While periods of lockdown and strict social distancing clearly reduce the spread of the virus, becoming frailer because of lockdown may mean that older adults could be more vulnerable to serious effects of the virus during these periods. There is an important need for strategies to help in reducing frailty in older adults while such restrictions are in place;

(ii) Regular exercise during lockdown can have beneficial long-term effects aiding recovery once restrictions relax. Helping older adults, especially those living with frailty, to remain physically active during periods of high restrictions should be an important consideration going forward.

In conclusion, lockdowns, social distancing restrictions and pauses in healthcare accessibility could have specific negative consequences for older people, particularly those living with long-term conditions that need ongoing treatment and support. While lockdowns protect the most vulnerable, these studies suggest factors that could lessen negative impacts of future lockdowns. Maintaining physical activity, supporting a sense of control and a supportive environment, as well as providing access to services such as GP or outpatient appointments during periods of lockdown are shown to be key priorities.

\section{ORCID iDs}

Carol Holland (1) https://orcid.org/0000-0001-7109-6554 lan Garner (1) https://orcid.org/0000-0002-9349-5377 Jane Simpson (1) https://orcid.org/0000-0001-5071-4077 Fiona Eccles (1) https://orcid.org/0000-0003-1484-2703 Esperanza Navarro Pardo (1) https://orcid.org/0000-0002-9355-2909 Calum Marr (10) https://orcid.org/0000-0003-3878-9505 Sandra Varey (1) https://orcid.org/0000-0002-6148-6425

\section{References}

1. World Health Organization. Pulse survey on continuity of essential health services during the COVID-19 pandemic: Interim report, 27 August 2020. https://www.who.int/publications/i/item/WHO-2019nCoV-EHS_continuity-survey-2020.1. Accessed November 6, 2021.

2. Woolf SH, Chapman DA, Sabo RT, Weinberger DM, Hill L. Excess deaths from COVID-19 and other causes, March-April 2020. JAMA. 2020;324(5):510-513. doi:10.1001/jama.2020.11787

3. Kontopantelis E, Mamas MA, Deanfield J, Asaria M, Doran T. Excess mortality in England and Wales during the first wave of the COVID-19 pandemic. JEpidemiol Community Health. 2021;75(3):213-223. doi:10. 1136/jech-2020-214764

4. Kondilis E, Tarantilis F, Benos A. Essential public healthcare services utilization and excess non-COVID-19 mortality in Greece. Public Health. 2021;198:85-88. doi:10.1016/j.puhe.2021.06.025
5. Department of Health. Long Term Conditions Compendium of Information, Third Edition. https://assets.publishing.service.gov.uk/ government/uploads/system/uploads/attachment_data/file/216528/ dh_134486.pdf. Accessed November 6, 2021.

6. Levin AT, Hanage WP, Owusu-Boaitey N, Cochran KB, Walsh SP, Meyerowitz-Katz G. Assessing the age specificity of infection fatality rates for COVID-19: Systematic review, meta-analysis, and public policy implications. Eur J Epidemiol. 2020;35(12):1123-1138. doi:10.1007/ s10654-020-00698-1

7. Atkins JL, Masoli JAH, Delgado J, et al. Preexisting comorbidities predicting COVID-19 and mortality in the UK biobank community cohort. J Gerontol A Biol Sci Med Sci. 2020;75(11):2224-2230. doi:10.1093/gerona/glaa183

8. Onder G, Rezza G, Brusaferro S. Case-fatality rate and characteristics of patients dying in relation to COVID-19 in Italy. JAMA. 2020;323(18): 1775-1776. doi:10.1001/jama.2020.4683

9. Clegg A, Young J, lliffe S, Rikkert MO, Rockwood K. Frailty in elderly people. Lancet. 2013;381(9868):752-762. doi:10.1016/S0140-6736 (12)62167-9

10. Hewitt J, Carter B, Vilches-Moraga A, et al. The effect of frailty on survival in patients with COVID-19 (COPE): A multicentre, European, observational cohort study. Lancet Public Health. 2020;5(8):e444-e451. doi:10.1016/S2468-2667(20)30146-8

11. Petermann-Rocha F, Hanlon P, Gray SR, et al. Comparison of two different frailty measurements and risk of hospitalisation or death from COVID-19: Findings from UK Biobank. BMCMed. 2020;18(1):355. doi:10.1186/s12916-020-01822-4

12. Apóstolo, J, Cooke R, Bobrowicz-Campos E, et al. Effectiveness of interventions to prevent pre-frailty and frailty progression in older adults: A systematic review. JBI Database System Rev Implement Rep. 2018;16(1):140-232. doi:10.11124/JBISRIR-2017-003382

13. Gale CR, Westbury L, Cooper C. Social isolation and loneliness as risk factors for the progression of frailty: The English Longitudinal Study of Ageing. Age Ageing. 2018;47(3):392-397. doi:10.1093/ageing/afx188

14. Oliveira JS, Pinheiro MB, Fairhall N, et al. Evidence on physical activity and the prevention of frailty and sarcopenia among older people: A systematic review to inform the World Health Organization physical activity guidelines. J Phys Act Health. 2020;17(12):1247-1258. doi:10.1123/jpah.2020-0323

15. Rodríguez-Gonzalez R, Facal D, Martinez-Santos AE, Gandoy-Crego M. Psychological, social and health-related challenges in Spanish older adults during the lockdown of the COVID-19 first wave. Front Psychiatry. 2020;11:588949. doi:10.3389/fpsyt.2020.588949

16. Simpson J, Zarotti N, Varey S, et al. 'It's a double whammy': A qualitative study of illness uncertainty in individuals with Parkinson's disease in the context of COVID-19. Chronic IIIn. 2021;17423953211043101. doi:10.1177/17423953211043101

17. Hansen BS, Rørtveit K, Leiknes I, et al. Patient experiences of uncertainty: A synthesis to guide nursing practice and research. J Nurs Manag. 2012;20(2):266-277. doi:10.1111/j.1365-2834.2011.01369.x

18. Simpson J, Eccles FJR, Doyle C. The impact of coronavirus restrictions on people affected by Parkinson's: The findings from a survey by Parkinson's UK. Lancaster, UK: Lancaster University; 2020. https://www. parkinsons.org.uk/news/how-have-coronavirus-covid-19-restrictionsimpacted-people-affected-parkinsons. Accessed November 6, 2021.

19. Garner IW, Burgess AP, Holland CA. Developing and validating the Community-Oriented Frailty Index (COM-FI). Arch Gerontol Geriatr. 2020;91:104232. doi:10.1016/j.archger.2020.104232

20. National Health Service. Who is at high risk from coronavirus (clinically extremely vulnerable). https://www.nhs.uk/conditions/coronavirus-covid-19/people-at-higher-risk/who-is-at-high-risk-from-coronavirus-clinically-extremely-vulnerable. Accessed November 6, 2021. 